\title{
COMBATE DE Panicum zizanoides H.B.K. y Paspalum conjugatum Beg. EN BANANO (Musa AAA) EN EL TRÓPICO HÚMEDO DE COSTA RICA ${ }^{1}$
}

\author{
Luis Acosta², Renán Agüero ${ }^{2}$
}

\begin{abstract}
RESUMEN
Combate de Panicum zizanoides H.B.K. y Paspalum conjugatum Beg. en banano (Musa AAA) en el trópico húmedo de Costa Rica. Se estudió el impacto de varios métodos químicos y físicos de manejo, sobre poblaciones de $P$. $z i$ zanoides y $P$. conjugatum. En una plantación de banano de 10 años de establecida se aplicaron seis tratamientos a saber: $\mathrm{T}_{1}=$ testigo libre crecimiento; $\mathrm{T}_{2}=$ chapeas mensuales; $\mathrm{T}_{3}=$ control con glifosato cada dos meses; $\mathrm{T}_{4}=$ controles mensuales con paraquat; $\mathrm{T}_{5}=$ control con glufosinato cada dos meses y $\mathrm{T}_{6}=$ control mixto. En las rodajas a los 75 días, las poblaciones de $P$. zizanoides con respecto a $\mathrm{T}_{1}$ se redujeron en un $89,93,79$ y $68 \%$ para $\mathrm{T}_{3}, \mathrm{~T}_{4}, \mathrm{~T}_{5}, \mathrm{~T}_{6}$ respectivamente, mientras que en las hileras se redujeron en un $80 \%$ para $\mathrm{T}_{4}$. A los 75 días en las rodajas las poblaciones de $P$. conjugatum se redujeron en $78,91,95,99$ y $75 \%$ para $\mathrm{T}_{2}, \mathrm{~T}_{3}, \mathrm{~T}_{4}, \mathrm{~T}_{5}$ y $\mathrm{T}_{6}$ respectivamente (Duncan; $\mathrm{p}<0,05$ ). Los tratamientos con herbicidas y control mixto redujeron las poblaciones de estas especies en los hábitats estudiados, mientras que en las parcelas con chapea y con testigos a libre crecimiento, las poblaciones de estas especies se mantuvieron estables en el tiempo.
\end{abstract}

\begin{abstract}
Control of Panicum zizanoides H.B.K. and Paspalum conjugatum Beg. under several strategies of management in banana (Musa AAA) plantation. The objective of this study was to determine the effect of several management strategies on the populations of these species. A ten-year-old banana plantation was chosen for evaluation with the following treatments: $\mathrm{T}_{1}=$ untreated control; $\mathrm{T}_{2}=$ physical control, $\mathrm{T}_{3}=$ successive sprays with glyphosate, $\mathrm{T}_{4}=$ successive sprays with paraquat, $\mathrm{T}_{5}=$ successive sprays with gluphosinate and $\mathrm{T}_{6}=$ mixed control based on weed sampling. Populations of $P$. zizanoides in rows were reduced $89,93,79$ and $68 \%$ for $\mathrm{T}_{3}, \mathrm{~T}_{4}, \mathrm{~T}_{5}, \mathrm{~T}_{6}$ respectively compared with $\mathrm{T}_{1}$. In the area next to the banana plant, populations of $P$. conjugatum were reduced by $78,91,95,99$ and $75 \%$ for $\mathrm{T}_{2}$, $\mathrm{T}_{3}, \mathrm{~T}_{4}, \mathrm{~T}_{5}$ and $\mathrm{T}_{6}$ respectively (Duncan; $\mathrm{p}<0.05$ ). The results indicate that treatments with herbicides and mixed control reduce the populations of these species, while populations of the untreated and physical control plots remained constant in time.
\end{abstract}

\section{INTRODUCCIÓN}

Panicum zizanoides H.B.K. y Paspalum conjugatum Beg. (Poaceacea), son dos de las principales malezas asociadas al agroecosistema de banano en el trópico húmedo de Costa Rica. Estas son especies estoloníferas perennes, nativas de América tropical y fueron registradas en 30 países y asociadas a más de 25 cultivos (Holm et al. 1977). P. conjugatum es una de las malezas más problemática en América, así como en el oeste de Africa y el sureste de Asia. En Hawaii se informó que $P$. conjugatum puede crecer hasta 1800 $\mathrm{msnm}$. Debido a su rápida propagación asexual (a través de estolones), forma una gran biomasa que puede

\footnotetext{
1 Recibido para publicación el 19 de marzo de 2002. Investigación financiada por la Universidad de Costa Rica, Laboratorio de malezas- LAMA, MONSANTO y CORBANA.

2 Candidato a Doctorado-Universidad de Costa Rica/ Coordinador de Proyectos, Prefectura, Tarija- Bolivia, luistarija@yahoo.com

3 LAMA-CIPROC. Facultad de Ciencias Agroalimentarias, Universidad de Costa Rica, raguero@cariari.ucr.ac.cr
} 
llegar a eliminar plántulas de árboles y arbustos de todo tipo (Gómez 1984). Es una planta que tolera ambientes sombreados por lo que puede sobrevivir en agroecosistemas de porte alto como el banano. También puede crecer en asociación con poblaciones de Panicum zizanoides en suelos pobres y ácidos. Poco es conocido acerca de la biología de esta especie. En algunas regiones como Filipinas se indicó que puede crecer y florecer todo el año, una planta puede llegar a producir hasta 1.500 semillas. Esta especie se considera como una de las principales malezas en piña, café, caucho, palma aceitera, té y cacao. También se mencionó como una de las principales malezas asociadas al cultivo del banano, en Filipinas, mientras que en Malasia fue la principal en cítricos y papaya. En Australia se la reporta en pasturas. En Trinidad y en México en caña de azúcar, cacao y yuca. $P$. conjugatum es una especie hospedera del hongo Sorosporium paspali McAlp. y del virus que produce mosaico en la caña de azúcar (Holm et al. 1977). Los nombres comunes de esta especie difieren entre países; en Australia se la denomina zacate amargo, grama (Bolivia y Brasil), horquetilla (Colombia y Costa Rica), zacate hilo (Hawaii), Assamese (India ), zacate búfalo (Malasia), pata de conejo (Mexico), tororuco, (Perú), ya-hey (Tailandia), paspalum amargo (Estados Unidos) y paja mala en Venezuela.

Existe poca literatura de $P$. zizanoides. Estudios preliminares acerca de su biología, la señalan como una planta monocotiledónea, herbácea, puede llegar hasta $70 \mathrm{~cm}$ de altura tallo culmoso decumbente estoloníferas hojas lanceoladas envainadoras y alternas. Es una especie que se propaga por semilla y vegetativamente por estolones y trozos de tallos que presentan sus nudos. Acosta y Aguiero (2001) mencionan a $P$. zizanoides como una de las principales malezas en el cultivo de banano en el trópico húmedo de Costa Rica, e indican que esta especie puede presentarse con densidades promedios de 79 individuos $/ \mathrm{m}^{2}$. Terry (1996) señala que malezas del género Panicum son problemáticas en el cultivo de banano y plátanos e indica que malezas de hoja pueden servir como coberturas para evitar la erosión del suelo.

Para desarrollar una estrategia de control en un determinado agroecosistema se requieren conocimientos básicos para entender mejor la influencia de los factores bióticos y abióticos que regulan el comportamiento de las poblaciones de malezas asociadas a éste. Estos conocimientos facilitarán la predicción de los efectos en el cultivo de las estrategias de combate de malezas que se adopten. El uso de productos químicos para el manejo de las poblaciones de malezas debe estar orientado a reducir la infestación de las mismas hasta un nivel que no cause daños económicos y no pretender una erradicación total. Se deben evitar los efectos se- cundarios indeseables de los productos químicos sobre todo cuando se usen dosis superiores a las normalmente recomendadas; en ocasiones estos efectos pueden consistir en problemas de fitotoxicidad para el cultivo de interés, efectos residuales en el suelo y efectos directos en la salud humana. Así Akobundu (1987) y Alstrom (1990) proponen que la inclusión del uso de herbicidas como un componente menor de los esquemas de control de malezas en la agricultura de los países tropicales, que sólo deben ser utilizables para problemas específicos y no como práctica habitual. Estos autores indican que la recomendación del uso de un determinado herbicida debe estar basado en los resultados de efectividad técnica y económica derivado de una evaluación previa a nivel de campo.

El objetivo de este estudio fue determinar el efecto de las estrategias de manejo con métodos químicos y físicos sobre las poblaciones de Panicum zizanoides y Paspalum conjugatum en el agroecosistema del banano. Con esta información se pretende desarrollar estrategias de manejo de estas especies más racionales.

\section{MATERIALES Y MÉTODOS}

El presente estudio se desarrolló durante un periodo de dos años (2000-2001). El mismo fue realizado en la finca San Pablo de la Corporación Bananera Nacional (Cantón Siquirres, Distrito de Pacuarito), ubicada en 28 Millas de la provincia de Limón, Costa Rica. Según el sistema de zonas de vida de Holdridge (1979) esta región se clasifica como bosque tropical húmedo, con una precipitación anual que fluctúa entre los 3.500 y $4.000 \mathrm{~mm}$. La temperatura promedio anual es de 25 ${ }^{\circ} \mathrm{C}$ y la humedad relativa promedio del $85 \%$. Los suelos donde se estableció el experimento corresponden a la clase III, IV y V, mientras que de acuerdo con uso potencial corresponden a las clases II y III. Estos suelos tienen una textura que varía de franco a franco arcillo limoso.

\section{Metodología}

El experimento se ubicó en una plantación de banano var. Gran enano de 10 años de establecida. Se seleccionó una superficie de $8400 \mathrm{~m}^{2}$, en la cual se estableció un diseño de bloques completos al azar con tres repeticiones distribuidas en forma aleatoria. La unidad experimental consistió de $400 \mathrm{~m}^{2}$ con 65 plantas de banano. En cada unidad experimental se definieron al azar, tres puntos de observación en la rodaja y tres entre las hileras, constituyendo un total de 104 puntos de observación. Estos puntos de observación fueron 
definidos como puntos de muestreo fijos, los mismos se identificaron con estacas. Seis tratamientos fueron evaluados en este experimento a saber: $\mathrm{T}_{1}=$ testigo libre crecimiento; $\mathrm{T}_{2}=$ control con chapias mensuales; $\mathrm{T}_{3}=$ control con glifosato cada dos meses; $\mathrm{T}_{4}=$ controles mensuales con paraquat; $\mathrm{T}_{5}=$ control con glufosinato cada dos meses y $\mathrm{T}_{6}=$ control mixto con chapea, glifosato y glufosinato cada dos meses.

Para los tratamientos con herbicidas se utilizó una bomba de espalda de 16 litros con boquilla Tee jet 8002 . Las dosis de los productos aplicados fueron establecidas con base en las recomendaciones emitidas por las casas comerciales representadas por cada uno, a saber: paraquat (Gramoxone-200 g/l; al 0,75\% v/v) = $120 \mathrm{ml} / 16 \mathrm{li}$ tros de agua; glufosinato (Finale-150 g/l; al $1 \% \mathrm{v} / \mathrm{v}$ ) con una dosis de $160 \mathrm{ml} / 16$ litros de agua y glifosato (Ranger Plus-680 g/kg al 0,687 \% p/v) con una dosis de $110 \mathrm{~g} / 16$ litros de agua. El volumen de aplicación osciló entre 200 y 250 l/ha, dependiendo de la densidad y el estado de las malezas en cada aplicación. Para la chapea se utilizó un machete común de $80 \mathrm{~cm}$ de longitud.

\section{Cuantificación de la vegetación predominante}

Se realizaron recuentos del número de individuos de Paspalum conjugatum y Panicum zizanoides presentes en los puntos de muestreo que se definieron en las rodajas y entre las hileras del banano, estos recuentos se hicieron antes del inicio de los tratamientos y a los 75, 150, 225 y 300 días después del inicio del estudio. Para tal efecto se utilizó un marco metálico dividido en cuadrados de $10 \mathrm{~cm}^{2}$ de área, el mismo fue colocado en cada estación de muestreo ubicado entre las hileras (hábitat 1) y otro marco de madera construido en forma de media luna de $0,60 \mathrm{~m}^{2}$ fue colocado al azar en la rodaja frente al hijo de espada (hábitat 2). Una vez identificadas las especies, se procedió a realizar el respectivo conteo de individuos en cada sitio.

Los datos se analizaron estadísticamente mediante el programa SAS, según el diseño experimental y el modelo matemático correspondiente. El análisis de variación se realizó para cada una de las especies y para comparar medias entre tratamientos se utilizó la prueba de rango múltiple de Duncan al 5\% de probabilidad.

\section{RESULTADOS Y DISCUSIÓN}

\section{Paspalum conjugatum Beg.}

En la primera evaluación realizada antes de la primera aplicación de los tratamientos no se obtuvieron diferencias significativas en la densidad de plantas de
$P$. conjugatum entre los sitios de muestreo. En la evaluación realizada a los 75 días después de la aplicación de los tratamientos, la densidad de plantas $/ \mathrm{m}^{2}$ difirió entre métodos físicos y químicos. En el hábitat entrelíneas después de los tratamientos se presentaron dos grupos a saber: El testigo a libre crecimiento, control con chapea y control combinado resultaron similares entre sí, mientras que los tratamientos con glifosato, paraquat y glufosinato redujeron significativamente las poblaciones de esta especie en relación al testigo. También a los 75 días, las poblaciones de $P$. conjugatum se redujeron en un 96, 95 y $78 \%$ con los tratamientos $\mathrm{T}_{3}$, $\mathrm{T}_{4} \mathrm{y}_{5}$ respectivamente. Estos porcentajes de control mantuvieron rangos similares en el tiempo con relación al testigo. Así a los 300 días las poblaciones de esta especie en este mismo hábitat disminuyeron un 99, 68 y $46 \%$ para los tratamientos con glifosato, paraquat y glufosinato, respectivamente. En el testigo a libre crecimiento las poblaciones de esta especie se mantuvieron iguales entre sí en las distintas fechas de evaluación (Cuadro 1a).

En el Cuadro 1b se indica la densidad de plantas $/ \mathrm{m}^{2}$ de $P$. conjugatum en la rodaja. Se observa que esa densidad de plantas fue inferior con relación al hábitat entre las hileras $\left(\mathrm{H}_{1}\right)$. Al respecto Acosta y Agüero (2001) indican que el número de individuos de esta especie en una plantación de banano fueron significativamente inferiores en el hábitat de las rodajas. Estos resultados se los podría atribuir a la sensibilidad de esta especie a los disturbios que se realizan en la rodaja, durante las operaciones de manejo del cultivo como la fertilización deshija y cosecha. En la evaluación realizada a los 75 y 150 días se presentaron dos grupos diferenciados, el testigo y el resto de los tratamientos. A los 150 días las poblaciones de esta especie disminuyeron 84, 99, 92, 96 y 96 $\%$ para $\mathrm{T}_{2}, \mathrm{~T}_{3}, \mathrm{~T}_{4}, \mathrm{~T}_{5}$ y $\mathrm{T}_{6}$ respectivamente, en relación al testigo.

En la evaluación realizada a los 225 días, se obtuvieron diferencias entre el testigo, el paraquat y el control mixto no así para los demás tratamientos cuando son comparados con el testigo (Cuadro 1b). La no diferencia entre el testigo glifosato y glufosinato, podría atribuirse al efecto de la luz, al reactivar las semillas latentes en el banco, cuando quedan los suelos deprovistos de vegetación por el uso intensivo de herbicidas. Al respecto Harper (1977) y Zelaya (1997) sostienen que un pulso de luz puede romper la latencia de las semillas enterradas y producirse una masiva germinación de semillas presentes en el banco.

Los resultados indican que tratamientos con glifosato, paraquat, glufosinato y controles mixtos tienen efecto en reducir las poblaciones de esta especie. Así Terry (1996) indica que las malezas anuales y perennes 
Cuadro 1. Densidad de plantas $/ \mathrm{m}^{2}$ de Paspalum conjugatum bajo diferentes estrategias de manejo en dos hábitats. Finca San Pablo de CORBANA. Siquirres, Limón, Costa Rica. 2000-2001.

a) hábitat entre línea

\begin{tabular}{lrrrrr}
\hline \multicolumn{1}{c}{ Tratamientos } & \multicolumn{5}{c}{ Evaluaciones } \\
& 0 días & 75 días & 150 días & 225 días & 300 días \\
\hline Testigo $\left(\mathrm{T}_{1}\right) 39,67 \mathrm{a}$ & & $26,00 \mathrm{a}$ & $41,33 \mathrm{a}$ & $52,78 \mathrm{a}$ & $25,67 \mathrm{a}$ \\
Chapea $\left(\mathrm{T}_{2}\right)$ & $17,33 \mathrm{a}$ & $23,67 \mathrm{a}$ & $21,67 \mathrm{ab}$ & $57,67 \mathrm{a}$ & $38,67 \mathrm{a}$ \\
Glifosato $\left(\mathrm{T}_{3}\right)$ & $24,33 \mathrm{a}$ & $1,00 \mathrm{~b}$ & $2,33 \mathrm{~b}$ & $0,67 \mathrm{~b}$ & $0,33 \mathrm{~b}$ \\
Paraquat $\left(\mathrm{T}_{4}\right)$ & $25,67 \mathrm{a}$ & $4,33 \mathrm{~b}$ & $7,33 \mathrm{~b}$ & $10,17 \mathrm{~b}$ & $8,16 \mathrm{ab}$ \\
Glufosinato $\left(\mathrm{T}_{5}\right)$ & $14,00 \mathrm{a}$ & $5,67 \mathrm{~b}$ & $7,67 \mathrm{~b}$ & $7,89 \mathrm{~b}$ & $13,89 \mathrm{ab}$ \\
Control mixto $\left(\mathrm{T}_{6}\right)$ & $39,67 \mathrm{a}$ & $55,00 \mathrm{a}$ & $33,00 \mathrm{a}$ & $42,11 \mathrm{a}$ & $21,22 \mathrm{a}$ \\
\hline
\end{tabular}

Letras iguales a lo largo de cada columna no difieren estadísticamente entre sí (Duncan; $\mathrm{P}<0,05)$.

b) hábitat rodaja

\begin{tabular}{lrrrrr}
\hline \multicolumn{1}{c}{ Tratamiento } & \multicolumn{5}{c}{ Evaluaciones } \\
& 0 días & 75 días & 150 días & 225 días & 300 días \\
\hline Testigo $\left(\mathrm{T}_{1}\right)$ & $20,00 \mathrm{a}$ & $45,67 \mathrm{a}$ & $47,33 \mathrm{a}$ & $65,00 \mathrm{a}$ & $36,19 \mathrm{a}$ \\
Chapea $\left(\mathrm{T}_{2}\right)$ & $2,67 \mathrm{a}$ & $9,67 \mathrm{~b}$ & $7,33 \mathrm{~b}$ & $29,33 \mathrm{ab}$ & $14,27 \mathrm{ab}$ \\
Glifosato $\left(\mathrm{T}_{3}\right)$ & $18,33 \mathrm{a}$ & $3,67 \mathrm{~b}$ & $0,33 \mathrm{~b}$ & $17,80 \mathrm{ab}$ & $1,11 \mathrm{~b}$ \\
Paraquat $\left(\mathrm{T}_{4}\right)$ & $5,67 \mathrm{a}$ & $2,33 \mathrm{~b}$ & $3,33 \mathrm{~b}$ & $2,22 \mathrm{~b}$ & $5,0 \mathrm{~b}$ \\
Glufosinato $\left(\mathrm{T}_{5}\right)$ & $3,67 \mathrm{a}$ & $0,33 \mathrm{~b}$ & $1,67 \mathrm{~b}$ & $20,00 \mathrm{ab}$ & $2,23 \mathrm{~b}$ \\
Control mixto $\left(\mathrm{T}_{6}\right)$ & $13,33 \mathrm{a}$ & $11,33 \mathrm{ab}$ & $1,67 \mathrm{~b}$ & $8,89 \mathrm{~b}$ & $12,52 \mathrm{~b}$ \\
\hline
\end{tabular}

Letras iguales a lo largo de cada columna no difieren estadísticamente entre sí (Duncan; $\mathrm{P}<0,05)$.

en crecimiento activo pueden ser controladas con un tratamiento de posemergencia de glifosato con dosis de 1-3 kg ia/ha, Lee y Ngim (1997) indican que herbicidas como paraquat, glufosinato de amonio y glifosato reducen la poblaciones de Ottochloa nodosa, Paspalum conjugatum e Imperata cinlindrica.

\section{Panicum zizanoides H.B.K.}

Al igual que $P$. conjugatum, un análisis individual por hábitat fue realizado para cada fecha de evaluación. Para la evaluación realizada antes de la interferencia, no se obtuvo diferencias entre poblaciones de esta especie en los diferentes sitios de muestreo en $\mathrm{H}_{1}$ (Figura 1). Sin embargo a los 75 y 150 días después de los tratamientos diferencias entre el testigo a libre crecimiento y paraquat fueron observadas (Duncan, $\mathrm{p}<0,05)$. A los 225 días se presentó dos grupos, un grupo identificado por el testigo, chapea y el control mixto y el otro por los tratamientos de glifosato, paraquat y glufosinato (Figura 1). A los 225 y 300 días las diferencias fueron mas notorias en los tratamientos químicos comparado con el resto de los tratamientos. Así, a los
300 días las poblaciones de $P$. zizanoides se redujeron en un 90,93 y $83 \%$ para $\mathrm{T}_{3}, \mathrm{~T}_{4}$ y $\mathrm{T}_{5}$ respectivamente.

Las poblaciones de $P$. zizanoides presentes en las rodajas, respondieron marcadamente a los diferentes

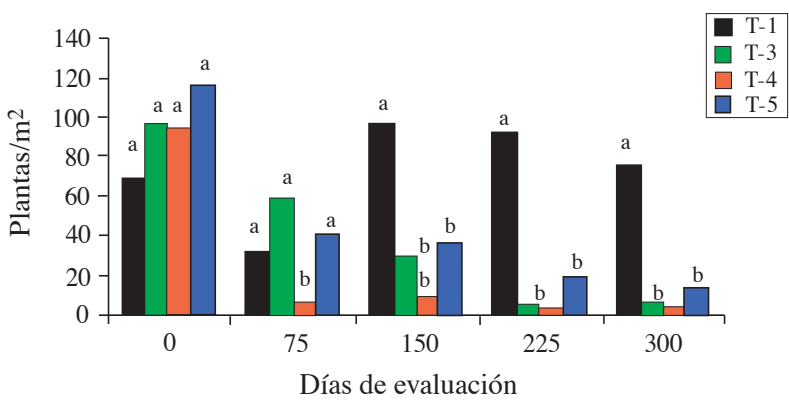

Figura 1. Poblaciones de P. zizanoides entre hileras bajo diferentes métodos de combate por un periodo de 300 días $\mathrm{T}-1=$ testigo libre crecimento; $\mathrm{T}-3=$ glifosato; $\mathrm{T}-4=$ paraquat; T-5 = glufosinato. Letras iguales entre barras no difieren estadísticamente entre sí, Duncan $\mathrm{p}<0,05$. Finca San Pablo de CORBANA. Siquirres, Limón, Costa Rica, 2000-2001. 
métodos de combate (Figura 2). La respuestas en este hábitat mostraron diferencias a partir de 75 días. Un grupo caracterizado por el testigo y el otro los tratamientos con herbicidas y controles mixtos. La reducción de las poblaciones de P. zizanoides comparado con el testigo a los 300 días fueron de 95, 90 y $99 \%$ para T3, T4 y T6 respectivamente.

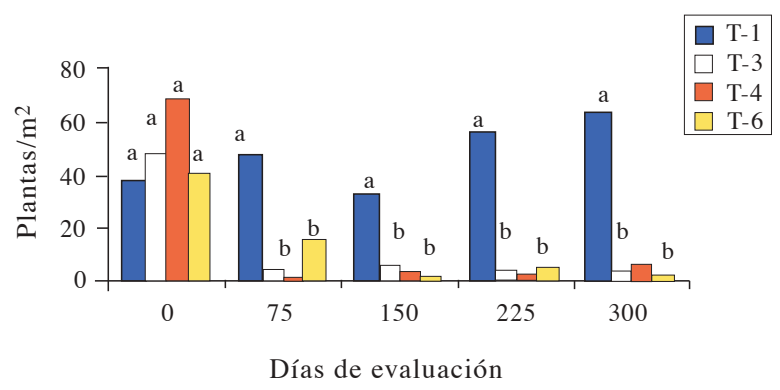

Figura 2. Poblaciones de P. zizanoides en rodajas bajo diferentes métodos de manejo, por un periodo de 300 días T$1=$ testigo libre crecimento; $\mathrm{T}-3=$ glifosato; $\mathrm{T}-4=$ paraquat; $\mathrm{T}-6=$ control mixto. Letras iguales entre barras no difieren estadísticamente entre sí, Duncan p<0,05. Finca San Pablo de CORBANA. Siquirres, Limón, Costa Rica, 2000-2001.

Aunque glifosato, paraquat, glufosinato y el control mixto redujeron las poblaciones de ambas poaceas, glifosato mostró el efecto más marcado. Sin embargo, las parcelas bajo control mixto, que contempló las zonas de mayor interferencia malezas-cultivo, con presencia de malezas en islas, mostraron los rendimientos de fruta de banano más altos (datos sin publicar), lo cual pareciera entonces indicativo de un tratamiento más racional.

Es de interés observar estos tratamientos por varios años, para comprobar si se establecen tendencias que definan con claridad las diferencias observadas hasta el momento, así como observar la dinámica poblacional de la flora espontánea. Las islas de malezas aunado a la dinámica de la hojarasca del propio cultivo, hace suponer que parcelas bajo tratamiento mixto observaran parámetros positivos de calidad de sitio, como la actividad de lombrices, baja escorrentía y baja compactación del suelo, al mismo tiempo que se minimiza la interferencia de las malezas con el banano y, por ende, se fomenta una alta producción de fruta.

\section{AGRADECIMIENTOS}

Al Dr. Primo Luis Chavarría por sus sugerencias para mejorar el presente artículo. A los trabajadores de la finca San Pablo de CORBANA, Costa Rica.

\section{LITERATURA CITADA}

ACOSTA, L.; AGÜERO R. 2001. Levantamiento taxonómico y densidad de la flora vascular asociada al agroecosistema banano (Musa AAA) en el trópico húmedo de Costa Rica. Presentado a la XLVII Reunion del Programa Cooperativo Centroamericano para el Mejoramiento de Cultivos (PCCMCA). San Jose Costa Rica.

AKOBUNDU, I.O. 1987. Weed science in the tropic-principles and practices. John Wiley \& Sons New York 522 p.

ALSTROM, S. 1990. Fundamentals of weed management in hot climate peasant agriculture. Crop Production Science 11, Uppsala, $271 \mathrm{p}$.

HARPER, J.L. 1977. Population biology of plants academic press London. New York. San Francisco 857 p.

HOLDRIDGE, L. 1979. Ecología basada en zonas de vida. San José, Costa Rica. IICA. 216 p.

HOLM, L.H.; PLUCKNETT, D.L.; PANCHO, J.V.; HERBERGER, J.P. 1977. The world's worst weeds distribution and biology. The East-West Center. University Press of Hawaii Honolulu. 609 p.

GÓMEZ, L.D. 1984. Las plantas acuáticas y anfibias de Costa Rica y Centroamérica. Editorial Universidad Estatal a Distancia San José Costa Rica 430 p.

LEE, L. J.; NGIM, J.; LEE, L. J. 1997. Evaluating non-selective herbicides for rainfast properties in Malaysia. Planter: 73: 65-68.

TERRY, P.J. 1996. Manejo de las malas hierbas en bananos y plátanos. In (Eds) LABRADA, R.; CASELEY, J.C.; PARKER, C. 1996. Manejo de malezas para países en desarrollo. Estudio FAO, Producción y Protección Vegetal 120: Italia, Roma 403 p.

ZELAYA, I. A. 1997. La reproducción de malezas. In Introducción a la Biología, Ecología y Manejo de Malezas Ed. Abelino Pitty Zamorano. Academic press 297 p. 